\title{
Gastro-oesophageal reflux and gastric aspiration in idiopathic pulmonary fibrosis patients
}

\author{
Edoardo Savarino ${ }^{1,2}$, Roberto Carbone ${ }^{3}$, Elisa Marabotto ${ }^{1}$, Manuele Furnari ${ }^{1}$, \\ Luca Sconfienza ${ }^{4,5}$, Massimo Ghio $^{6}$. Patrizia Zentilin ${ }^{1}$ and Vincenzo Savarino ${ }^{1}$
}

\begin{abstract}
Affiliations: 'Division of Gastroenterology, Dept of Surgical, Oncological and Gastroenterological Sciences, University of Padua, Padua, ${ }^{2}$ Division of Gastroenterology, Dept of Internal Medicine, University of Genoa, Genoa, ${ }^{3}$ Pneumology Unit, Regional Hospital of Aosta, Aosta, ${ }^{4}$ Servizio di Radiologia, IRCCS Policlinico San Donato, San Donato Milanese, ${ }^{5}$ Dipartimento di Scienze Biomediche per la Salute, Università degli Studi di Milano, San Donato Milanese, and ${ }^{6}$ Division of Clinical Immunology, Dept of Internal Medicine, University of Genoa, Genoa, Italy.
\end{abstract}

Correspondence: E. Savarino, Division of Gastroenterology, University of Genoa, Viale Benedetto XV 6, 16132 Genoa, Italy. E-mail: esavarinollibero.it

ABSTRACT The aim of the study was to characterise gastro-oesophageal reflux (GOR) in idiopathic pulmonary fibrosis (IPF).

40 consecutive IPF patients underwent pulmonary high-resolution computed tomography (HRCT) scan and impedance-pH monitoring while off antisecretory therapy. The presence of pulmonary fibrosis was assessed using validated HRCT scores. Reflux features included distal oesophageal acid exposure, number of acid/weakly acidic reflux episodes and their proximal migration. 40 consecutive patients with interstitial lung disease other than IPF (non-IPF patients) and 50 healthy volunteers were also enrolled.

IPF patients had significantly higher $(\mathrm{p}<0.01)$ oesophageal acid exposure (median (interquartile range (IQR)) 9.25 (4.7-15.4)\% versus $3.3(1.4-7.4) \%$ versus 0.7 (0.2-4.2)\%, number of acid (median (IQR) 45 (23-55) versus 32 (19-44) versus 18 (10-31)), weakly acidic (median (IQR) 34 (19-43) versus 21 (11-33) versus 18 (15-28)) and proximal reflux (median (IQR) 51 (26.5-65.5) versus 20 (9.5-34.5) versus 9 (5-20)) events compared to non-IPF patients and healthy volunteers, respectively. Pulmonary fibrosis HRCT scores correlated well with reflux episodes in both the distal $\left(r^{2}=0.567\right)$ and proximal $\left(r^{2}=0.6323\right)$ oesophagus. Patients with IPF had more bile acids and pepsin $(\mathrm{p}<0.03)$ in bronchoalveolar lavage fluid (BALF) $(62 \%$ and $67 \%$, respectively) and saliva $(61 \%$ and $68 \%$, respectively) than non-IPF patients $(25 \%$ and $25 \%$ in BALF, and $33 \%$ and $36 \%$, respectively, in saliva) and controls ( $0 \%$ and $0 \%$ in BALF and saliva, respectively).

Acid GOR is common in IPF, but weakly acidic GOR may also occur. Patients with IPF had a risk of pulmonary aspiration of gastric contents. Outcome studies with intense antireflux therapy are needed.

@ERSpublications

Frequent acid/weakly acidic gastro-oesophageal reflux and high risk of pulmonary aspiration of gastric contents in IPF http://ow.ly/nSNv8

Received: June 302012 | Accepted after revision: Feb 122013 | First published online: March 072013

Conflict of interest: None declared.

Copyright @ERS 2013 


\section{Introduction}

Idiopathic pulmonary fibrosis (IPF) is a chronic fibrotic lung disease with a median survival ranging from 2 to 3 years from diagnosis [1]. To date, no cure is available and treatment strategies show little effect. The mechanisms leading to IPF remain unknown. Gastro-oesophageal reflux (GOR) disease has been previously associated with a number of interstitial lung diseases (ILDs) [2-6].

Evidence from both animal [7] and human [8] studies support the concept that pulmonary fibrosis can occur after repeated tracheobronchial aspiration of small amounts of gastric contents over long periods of time (i.e. chronic microaspiration). Moreover, recent investigations have demonstrated disease stabilisation or delay of disease progression after medical or surgical treatment of GOR [9-11]. In particular, LEE et al. [11] showed that the use of GOR medications is associated with decreased radiologic fibrosis and is an independent predictor of longer survival time in patients with IPF.

Until recently, diagnosis and therapy of GOR has focused on gastric acid as the main monitoring parameter and treatment target. Recent studies highlighted that lung injury is independent of acidity and factors other than acid (i.e. foods, pepsin and bile acids) may be involved in its pathogenesis $[12,13]$. In particular, MERTENs et al. [13] showed that exposure of bronchial epithelial cells to gastric juice from patients on antisecretory therapy is able to induce high interleukin- 8 production, the most relevant cytokine for the acutephase response of inflammation. However, in IPF patients, GOR has previously been assessed by means of 24-h pH-metry that permits detection of acid reflux only. The recent use of impedance- $\mathrm{pH}$ monitoring allows us to detect both acid and weakly acidic GOR and to assess the extent of reflux into the proximal oesophagus $[14,15]$.

The aim of our study was to further explore the association of GOR and pulmonary fibrosis by prospectively assessing the prevalence of all kinds of reflux in a carefully selected, well-defined population of IPF patients and compare the findings to those of patients with ILD other than IPF and healthy volunteers.

\section{Patients and methods \\ Subjects}

Between November 2007 and October 2011, consecutive patients with a definite diagnosis of IPF were prospectively enrolled in the study. The diagnosis of IPF was based on the absence of an identifiable aetiology for ILD and a histopathological/radiological pattern of usual interstitial pneumonia on surgical lung biopsy and high-resolution computed tomography (HRCT) scans [1]. 40 consecutive patients with ILD other than IPF, referred for routine visits (independently of reported symptoms and primary disease type or stage) were also enrolled for comparison (participation rate of 92\%). They were found to have ascertained diseases associated with the development of pulmonary fibrosis: sarcoidosis $(\mathrm{n}=10)$, systemic lupus erythematosus $(n=6)$, mixed connective tissue disease $(n=14)$ and bronchiolitis obliterans organising pneumonia $(n=10)$, which were diagnosed according to characteristic histopathological findings on surgical lung biopsy or appropriate autoimmune markers and clinical presentations [16-19].

50 healthy volunteers without any disease or history of surgery were also enrolled for comparison.

The study protocol was approved by the local ethics committee and performed according to the Declaration of Helsinki. All participants gave written informed consent prior to being enrolled in the study.

\section{Study protocol}

All patients completed a structured questionnaire including patient demographics, history of pulmonary and reflux symptoms, medications and tobacco use. Then they underwent oesophageal and pulmonary investigations within 1 month of the date of diagnosis (defined as the date of initial visit) and saliva samples were collected for measuring bile acids and pepsin. Moreover, in patients who underwent bronchoscopy, bronchoalveolar lavage fluid (BALF) samples using standardised procedures were also collected for measuring bile acids and pepsin. All patients were asked to discontinue any medication that would influence oesophageal motility (i.e. nitrates, calcium antagonists, domperidone, benzodiazepines and metoclopramide) and acid suppressive therapy $\geqslant 30$ days before the start of the study.

\section{Oesophageal investigations}

Manometry testing

Oesophageal manometry was performed by means of multichannel intraluminal impedance-oesophageal manometry using a Koenigsberg 9-channel probe (Sandhill EFT; Sandhill Scientific Inc., Highlands Ranch, CO, USA). Testing methodology has been reported previously [20]. 
We measured lower oesophageal sphincter (LOS) pressure and relaxation, peak contraction amplitude, duration of contraction, coordination and propagation of velocity after swallows. Manometric patterns were reported according to international criteria [21].

\section{Impedance-pH monitoring}

The equipment used (Sleuth; Sandhill Scientific, Inc.) and testing methodology have been described previously [22]. Studies were performed off proton pump inhibitor treatment.

Data were downloaded and analysed using dedicated software (BioView Analysis; Sandhill Scientific Inc., Highlands Ranch, CO, USA) and subsequently reviewed manually by an experienced investigator blinded to the basal condition of the overall patients and healthy volunteers (E. Savarino). GOR episodes were classified as acid (nadir $\mathrm{pH}<4$ ), weakly acidic (nadir $\mathrm{pH} 4-7$ ) and weakly alkaline (nadir $\mathrm{pH}>7$ ) following established criteria [23].

Number and type of reflux episodes, acid exposure (reflux time (min) and reflux percentage time) and proximal extent (reflux reaching $15 \mathrm{~cm}$ above the LOS) were calculated.

Total distal oesophageal acid exposure $<4.2 \%$ over 24 h was considered normal $[24,25]$. Number of reflux episodes $<54$ was considered normal [26].

\section{Pulmonary investigations}

Pulmonary involvement was systematically investigated during the initial evaluation of IPF in all patients, by chest radiography, HRCT scan of the lungs and pulmonary function tests.

\section{Pulmonary function tests}

Forced vital capacity (FVC) and forced expiratory volume (FEV) curves were measured in a constantvolume plethysmograph (Sensor Medics 28000, Yorba Linda, CA, USA). A 10-s single-breath diffusing capacity of the lung for carbon monoxide (DLCO) test (Morgan, Chatham, UK) was carried out. Pulmonary function was considered abnormal if volumes were $<80 \%$ predicted values and/or when DLCO was $<75 \%$ pred $[27,28]$.

\section{Pulmonary HRCT}

High resolution scanning (CT Lightspeed; General Electric, Milwaukee, WI, USA) of the lungs was performed using standardised protocols to identify radiographic abnormalities related to IPF [1]. The testing methodology has been described previously [3].

HRCT scans were evaluated by two experienced radiologists, independently and in random order without knowledge of the patients' status. The overall interobserver agreement in assigning the fibrosis score was excellent $(\mathrm{K}=0.88)$. Any discrepancy in the assessment was discussed further and a consensus was reached. A semiquantitative analysis of the severity of fibrosis on HRCT was calculated by estimating the percentage of lung affected by fibrosis (i.e. reticular abnormality and/or honeycombing) to the nearest $5 \%$ in three zones for each lung, as previously described by BEST et al. [29]. These numbers were averaged to obtain a net radiologic fibrosis score.

\section{Monitoring of bile acids/pepsinogen in BALF and saliva}

Bile acids assay

BALF and saliva/sputum samples were analysed for the presence of total bile acids using a commercial assay (Bioquant, San Diego, CA, USA). The lowest level of accurate detection was $0.2 \mathrm{mmol} \cdot \mathrm{L}^{-1}[30]$.

Pepsin test

BALF and saliva/sputum samples were analysed for the presence of pepsin using a commercial enzymelinked immunoadsorbent assay (Peptest; RD Biomed Ltd, Hull, UK) [31].

\section{Saliva}

Bile acids and pepsin were measured in saliva samples collected under fasting condition in 38 IPF patients at the time of the first visit. Bile acids and pepsin were also measured in saliva from 36 patients with ILD other than IPF (sarcoidosis $(n=8)$, systemic lupus erythematosus $(n=5)$, mixed connective tissue disease $(n=13)$ and bronchiolitis obliterans organising pneumonia $(n=10)$ ) and from 50 healthy volunteers at the same time.

\section{Bronchoalveolar lavage}

Bronchoalveolar lavage was performed in a single subsegment of the right middle lobe or lingula, with $\geqslant 100 \mathrm{~mL}$ of sterile saline instilled. The BALF was recovered by gentle manual suction, kept on ice and 
TABLE 1 Demographic and clinical characteristics of idiopathic pulmonary fibrosis (IPF) patients, those with interstitial lung disease (ILD) other than IPF and healthy volunteers

IPF ILD other than IPF Healthy volunteers p-value

\begin{tabular}{|c|c|c|c|c|}
\hline Patients $\mathrm{n}$ & 40 & 40 & 50 & \\
\hline Female $\mathrm{n}$ & 18 & 21 & 27 & 0.676 \\
\hline Age years & $65(42-82)$ & $62(38-80)$ & $58(34-76)$ & 0.277 \\
\hline Body mass index $\mathrm{kg} \cdot \mathrm{m}^{-2}$ & $24(17-31)$ & $25(17-37)$ & $24(18-34)$ & 0.072 \\
\hline Alcohol use \% & 48 & 45 & 44 & 1 \\
\hline Surgical lung biopsies \% & 100 & 30 & NA & $<0.001$ \\
\hline Pulmonary symptoms ${ }^{\#} \%$ & 88 & 85 & NA & 1 \\
\hline FVC $\%$ & 55 (37-78) & $63(37-79)$ & 102 (87-110) & $<0.001$ \\
\hline FEV10 \% & $58(38-72)$ & $62(40-74)$ & $108(98-131)$ & $<0.001$ \\
\hline Radiological fibrosis score \% & 20 & 18 & NA & 0.0999 \\
\hline Oesophageal symptoms \% & 48 & 43 & NA & 0.8224 \\
\hline GOR medication ${ }^{+} \%$ & 40 & 43 & NA & 1 \\
\hline Drugs potentially influencing oesophageal motility $\%$ & 20 & 30 & NA & 0.4391 \\
\hline
\end{tabular}

Data are presented as median (interquartile range), unless otherwise stated. DLCO: diffusing capacity of the lung for carbon monoxide; FVC: forced vital capacity; FEV10: forced expiratory volume in $10 \mathrm{~s}$; GOR: gastro-oesophageal reflux; NA: not applicable. " ${ }^{\#}$ : i.e. cough and dyspnoea; ${ }^{\top}$ : i.e. heartburn and regurgitation; ${ }^{+}$: i.e. proton pump inhibitor or $\mathrm{H} 2$ blocker; $^{\S}$ : e.g. nitrates or calcium antagonists, domperidone, benzodiazepines or metoclopramide.

processed within $1 \mathrm{~h}$ of collection. Bile acid and pepsin were measured in BALF samples of 21 IPF patients obtained at the time of diagnosis during bronchoscopy. They were also measured in BALF of 20 patients with ILD other than IPF (10 sarcoidosis and 10 bronchiolitis obliterans organising pneumonia) and in 16 patients undergoing bronchoscopy for other diseases (non-ILD patients: 10 lung cancer and six chronic obstructive pulmonary disease).

\section{Statistical analysis}

Differences in proportions were compared using the Chi-squared or Fisher's exact test. As reflux parameters were not normally distributed, results are reported as median and interquartile range (IQR) and 95th percentile. Differences between groups were assessed using Kruskal-Wallis and/or Mann-Whitney tests. The correlation between the severity of pulmonary fibrosis and reflux parameters was calculated using Spearman correlation. The interobserver variability in grading fibrosis on HRCT was assessed by $\kappa$ statistics with linear weighting. For statistical significance $\alpha$ was set at 0.05 .

\section{Results}

\section{Patient characteristics}

40 patients with a definite diagnosis of IPF and 40 with pulmonary fibrosis other than IPF (non-IPF) were consecutively enrolled in the study. Detailed demographic and clinical characteristics of all patients and healthy volunteers are shown in table 1 . Patients with IPF had similar DLCO (48\% versus 50\%; $\mathrm{p}=0.1202)$,

TABLE 2 Manometric parameters of idiopathic pulmonary fibrosis (IPF) patients, those with interstitial lung disease (ILD) other than IPF and healthy volunteers

\begin{tabular}{|c|c|c|c|c|}
\hline & IPF & ILD other than IPF & Healthy volunteers & p-value \\
\hline Mean basal LOS pressure $\mathrm{mmHg}$ & $14.1(4-32.5)$ & $16.7(4-37.2)$ & $22(12-42)$ & $<0.001$ \\
\hline Mean proximal contraction amplitude $\mathrm{mmHg}$ & $92.4(40-190)$ & $84(35-170)$ & $94.7(35-170)$ & 0.441 \\
\hline Mean distal contraction amplitude $\mathrm{mmHg}$ & $90.6(10-210)$ & $79.9(10-210)$ & $85.36(30-190)$ & 0.439 \\
\hline Manometric hiatal hernia \% & 55 & 38 & 14 & $<0.001$ \\
\hline Mean basal UOS pressure $\mathrm{mmHg}$ & $60.1(38-100)$ & $62.4(35-110)$ & $64(35-110)$ & 0.787 \\
\hline
\end{tabular}

Data are presented as mean (interquartile range), unless otherwise stated. LOS: lower oesophageal sphincter; UOS: upper oesophageal sphincter. 
FEV (55\% versus $63 \% ; \mathrm{p}=0.2235)$, FVC $(58 \%$ versus $62 \% ; \mathrm{p}=0.0833)$ and radiologic fibrosis score $(20 \%$ versus $18 \% ; \mathrm{p}=0.0999)$ compared with non-IPF patients. Moreover, IPF patients tended to have a higher smoking history compared with non-IPF patients, but statistical significance was not reached $(\mathrm{p}=0.1759)$. All healthy volunteers had normal pulmonary (pulmonary function tests, chest radiographs and HRCT) and oesophageal (manometry and impedance-pH) investigations.

\section{Oesophageal manometric evaluation}

Patients with IPF had similar LOS resting pressure compared to non-IPF patients $(14.1 \mathrm{mmHg}$ versus $16.7 \mathrm{mmHg} ; \mathrm{p}=0.0999)$. Mean contraction amplitude in the distal and proximal oesophagus were slightly higher in IPF compared with non-IPF patients $(92.4 \mathrm{mmHg}$ versus $84 \mathrm{mmHg}$ and $90.6 \mathrm{mmHg}$ versus $79.9 \mathrm{mmHg}, \mathrm{p}=0.5285$ and $\mathrm{p}=0.2582$, respectively). No differences were found in terms of mean upper oesophageal sphincter basal pressure and prevalence of hiatal hernia between patients with IPF and non-IPF patients $(60.1 \mathrm{mmHg}$ versus $62.4 \mathrm{mmHg}$ and $55 \%$ versus $38 \%, p=0.9386$ and $\mathrm{p}=0.1782$, respectively). Moreover, IPF and non-IPF patients differed from healthy volunteers only in terms of mean LOS basal pressure and prevalence of hiatal hernia (table 2).

With regard to oesophageal motility patterns, no differences were found between IPF and non-IPF patients in terms of prevalence of normal peristalsis $(p=1)$, distal oesophageal spasm $(p=0.737)$, nutcracker oesophagus $(p=1)$, ineffective oesophageal motility $(p=1)$ and abnormally low LOS pressure $(p=0.6001)$.

\section{Oesophageal 24-h impedance-pH monitoring}

pH-metry data

Of 40 IPF patients, 33 (83\%) had an abnormal distal acid exposure, compared with 17 (43\%) out of 40 nonIPF patients $(\mathrm{p}<0.0001)$ (fig. 1). Percentage total, upright and recumbent time with $\mathrm{pH}<4$ was significantly higher in IPF compared with non-IPF patients and healthy volunteers (percentage total AET $9.25(4.7-15.4,25.6)$ versus $3.3(1.4-7.4,17.3)$ versus $0.7(0.2-4.1,4.2)$, for IPF patients, non-IPF patients and healthy volunteers, respectively; $\mathrm{p}<0.0001)$.

Impedance data

In IPF patients the total (both acid and weakly acidic) number of reflux episodes was higher (76 (43-96); 117) than that of non-IPF patients (47 (30.5-72); 104; p<0.0070) and healthy volunteers (32 (20-45); 55; $\mathrm{p}<0.0001$ ) (fig. 2). This was also the case when considering acid and weakly acidic reflux episodes separately $(\mathrm{p}<0.03)$.

More reflux episodes reached the proximal oesophagus (51 (26.5-65.5); 95) in IPF patients than non-IPF patients $(20.5$ (9.5-34.5); 62; $\mathrm{p}<0.0001)$ and healthy volunteers $(9(5-20) ; 32 ; \mathrm{p}<0.0001)$, as shown in figure 3. In addition, the percentage of total reflux episodes reaching the proximal measuring site was higher in IPF $(66 \%)$ than non-IPF patients $(42 \% ; \mathrm{p}<0.0001)$ and healthy volunteers $(31 \%$; $<<0.0001)$.

\section{Pulmonary aspiration of gastroduodenal contents}

Bile acids and pepsin in saliva

Saliva samples were collected in 38 IPF patients, 36 patients with ILD other than IPF and in 50 healthy volunteers. IPF patients had more bile acids and pepsin in saliva than non-IPF ones and healthy volunteers.

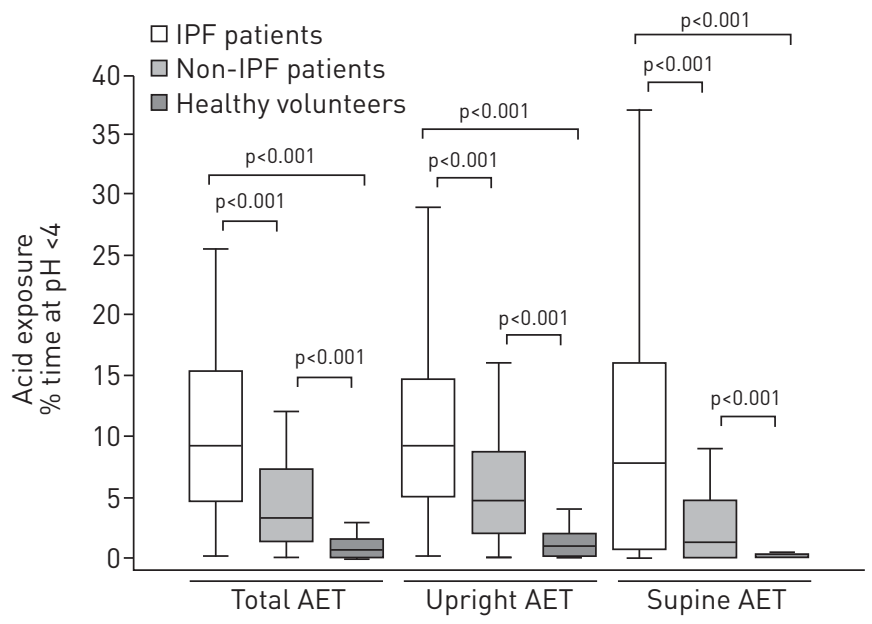

FIGURE 1 Median values of oesophageal acid exposure time (AET) in healthy volunteers $(n=50)$, patients with idiopathic pulmonary fibrosis (IPF) $(\mathrm{n}=40)$ and non-IPF patients $(n=40)$. Data are presented as median values, interquartile range and range. 
FIGURE 2 Number and types of gastrooesophageal reflux in healthy volunteers $(\mathrm{n}=50)$, patients with idiopathic pulmonary fibrosis (IPF) $(\mathrm{n}=40)$ and non-IPF patients $(\mathrm{n}=40)$. Data are presented as median values, interquartile range and range.

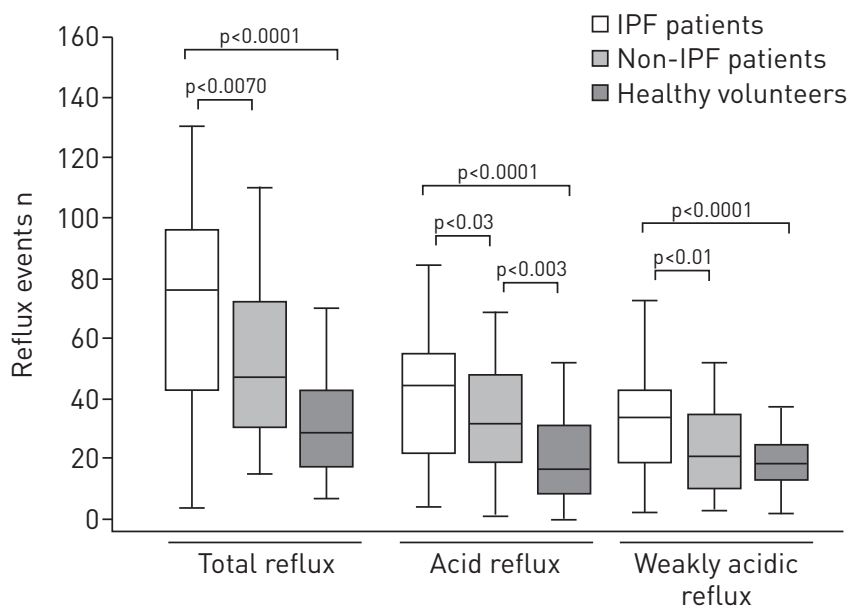

Bile acids were present in saliva of $23(61 \%)$ out of 38 IPF patients compared to $0(0 \%)$ out of 50 ( $\mathrm{p}<0.0001)$ healthy volunteers and $12(33 \%)$ out of $36(\mathrm{p}<0.0223)$ non-IPF patients. Pepsin was present in the saliva of 26 $(68 \%)$ out of 38 patients compared to $0(0 \%)$ out of $50(\mathrm{p}<0.0001)$ healthy volunteers and $13(36 \%)$ out of 36 $(\mathrm{p}=0.0099)$ non-IPF patients. The concentration of bile acids in saliva was significantly higher in patients with $\operatorname{IPF}\left(3.70(2.90-6.35) \mu \mathrm{mol} \cdot \mathrm{L}^{-1}\right)$ compared with non-IPF patients $\left(1.5(1.2-1.8) \mu \mathrm{mol} \cdot \mathrm{L}^{-1}\right)(\mathrm{p}<0.01)$. All IPF and non-IPF patients with bile acids or pepsin in saliva (92\% and $85 \%$, respectively) had abnormal impedance- $\mathrm{pH}$ test results (i.e. abnormal acid exposure time or abnormal number of reflux episodes).

\section{Bile acids and pepsin in BALF}

BALF samples were collected in 21 IPF patients, 20 patients with ILD other than IPF and in 16 patients undergoing bronchoscopy for other diseases. Patients with IPF had more bile acids and pepsin in BALF than the non-IPF ones and non-ILD patients. Bile acids were detected in BALF of $13(62 \%)$ out of 21 IPF patients compared with $0(0 \%)$ out of $16(\mathrm{p}<0.0001)$ non-ILD patients and in five $(25 \%)$ out of $20(\mathrm{p}=0.0278)$ non-IPF patients. Pepsin was present in BALF of $14(67 \%)$ out of 21 IPF patients compared with $0(0 \%)$ out of $16(\mathrm{p}<0.0001)$ non-ILD patients and $5(25 \%)$ out of $20(\mathrm{p}<0.0122)$ non-IPF patients. The concentration of bile acids in BALF was higher in IPF patients $\left(0.90(0.80-1.0) \mu \mathrm{mol} \cdot \mathrm{L}^{-1}\right)$ than in non-IPF patients $(0.50$ $\left.(0.40-0.60) \mu \mathrm{mol} \cdot \mathrm{L}^{-1}\right)(\mathrm{p}<0.01)$. All IPF and non-IPF patients with bile acids or pepsin in BALF $(93 \%$ and $80 \%$, respectively) had an abnormal impedance- $\mathrm{pH}$ test. Among the IPF patients, three patients were discrepant in terms of presence of pepsin and bile acids in BALF. Among the non-IPF patients, none was discrepant in relation to the presence of pepsin and bile acids in BALF.

FIGURE 3 Number of reflux episodes reaching the proximal oesophagus in healthy volunteers $(n=50)$, patients with idiopathic pulmonary fibrosis (IPF) $(\mathrm{n}=40)$ and non-IPF patients $(\mathrm{n}=40)$. Data are presented as median values, interquartile range and range.

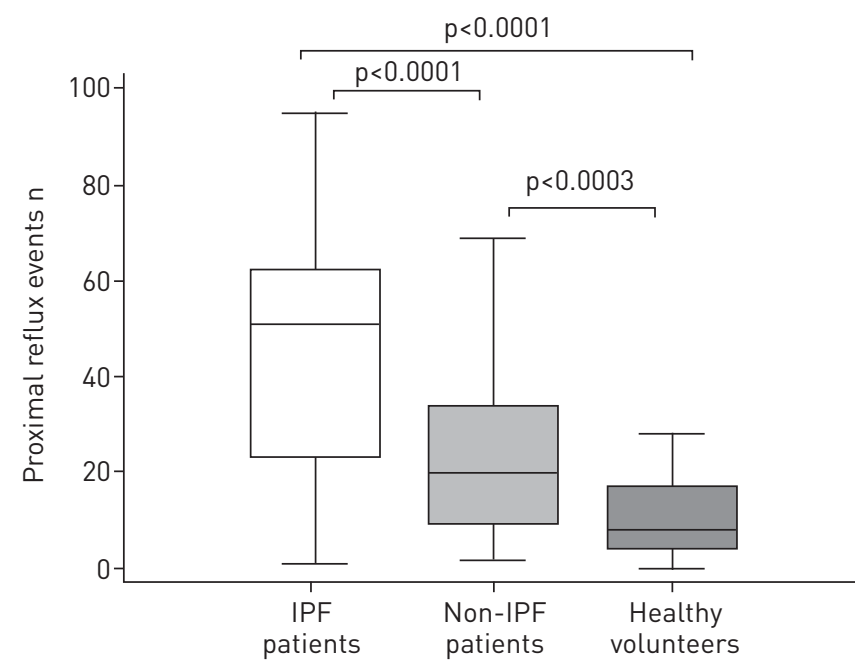


TABLE 3. Correlation between high-resolution computed tomography (HRCT) score and impedance-pH findings lacid exposure time and impedance-detected reflux episodes) with the presence/concentration of pepsin and bile acids in saliva and bronchoalveolar lavage fluid (BALF) in idiopathic pulmonary fibrosis patients

\begin{tabular}{|c|c|c|c|c|c|c|}
\hline & \multicolumn{2}{|c|}{ HRCT score } & \multicolumn{2}{|c|}{ Acid exposure time } & \multicolumn{2}{|c|}{ Total number of reflux episodes } \\
\hline Presence of bile acids in saliva & 0.4269 & $<0.001$ & 0.1493 & 0.017 & 0.5264 & 0.001 \\
\hline Concentration of bile acids in saliva & 0.2591 & 0.013 & 0.0129 & 0.606 & 0.0712 & 0.218 \\
\hline Presence of pepsin in BALF & 0.6033 & $<0.001$ & 0.2344 & 0.026 & 0.2845 & 0.013 \\
\hline
\end{tabular}

Correlation between HRCT score and impedance-pH findings loesophageal acid exposure time and reflux episodes) with the presence/concentration of pepsin and bile acids in saliva and BALF

In IPF patients there was a good correlation between degree of pulmonary fibrosis (HRCT score) and total number of reflux episodes in both distal $\left(\mathrm{r}^{2}=0.567, \mathrm{p}<0.001\right)$ and proximal $\left(\mathrm{r}^{2}=0.6323, \mathrm{p}<0.001\right)$ oesophagus. In contrast, in non-IPF patients a nonsignificant correlation was found between degree of pulmonary fibrosis and total number of reflux episodes in both distal $\left(\mathrm{r}^{2}=0.0955, \mathrm{p}=0.052\right)$ and proximal $\left(\mathrm{r}^{2}=0.0224, \mathrm{p}=0.356\right)$ oesophagus.

Detailed data on the correlation between HRCT score and impedance-pH parameters with presence/ concentration of gastric contents are summarised in table 3. A good correlation was found between HRCT score and presence of pepsin and bile acids in saliva $\left(\mathrm{r}^{2}=0.5260, \mathrm{p}<0.001\right.$ and $\mathrm{r}^{2}=0.4269, \mathrm{p}<0.001$, respectively) as well as in BALF $\left(\mathrm{r}^{2}=0.6033, \mathrm{p}<0.001\right.$ and $\mathrm{r}^{2}=0.4605, \mathrm{p}=0.001$, respectively). A poor but significant correlation was observed between HRCT score and the concentration of bile acids in saliva $\left(\mathrm{r}^{2}=0.2591, \mathrm{p}=0.013\right)$ and in BALF $\left(\mathrm{r}^{2}=0.3843, \mathrm{p}=0.024\right)$.

\section{Discussion}

Recent data have emphasised the role of GOR in the pathogenesis and potential management of IPF patients [9-13], but, although it is recognised that GOR is increased in IPF patients, its prevalence, characteristics and association with pulmonary aspiration of gastric contents have been poorly defined. In the current study, we investigated oesophageal motility, acid and weakly acidic reflux, proximal migration of refluxate, markers of gastric aspiration and their correlation with GOR and radiologic fibrosis, as well as the correlation between GOR and lung fibrosis in patients with IPF. We compared the results with those obtained from patients with ILD other than IPF and normal subjects. To our knowledge, this study is the first investigating the association of weakly acidic GOR with pulmonary findings in patients with IPF. We observed a higher frequency of GOR episodes (both acid and weakly acidic) and reflux episodes reaching the proximal oesophagus in patients with IPF compared to non-IPF patients, despite a similar oesophageal peristalsis profile at manometry testing. Moreover, we noted that the majority of IPF patients have risk of gastric aspiration (increased bile acids and pepsin in saliva) or definite gastric aspiration (bile acids or pepsin in BALF). Finally, we found a good correlation between the degree of pulmonary fibrosis and the severity of GOR, as well as between the degree of pulmonary fibrosis and the presence/concentration of gastric contents in the lungs. These findings, with the nonsignificant correlation between the degree of pulmonary fibrosis and the severity of GOR in non-IPF patients, suggest that patients with IPF have more severe GOR, potentially leading to more extensive lung damage and fibrosis progression.

To date, the mechanisms determining IPF are not clear. Current concepts implicate epithelial-fibroblast interactions as a result of repeated insults to the lung parenchyma by an unknown noxious stimulus. This prolonged stimulus would determine the development of pulmonary fibrosis over a long period of time [1]. Recent studies have suggested chronic microaspiration of gastric contents into the lungs as the trigger mechanism able to induce pulmonary parenchymal lesions, thus leading to the hypothesis that GOR therapy could improve symptoms and pulmonary function test parameters [3-6, 8-11]. This possibility has been recently emphasised, and at least in part confirmed, by the retrospective study by LeE et al. [11], who observed that the use of GOR medication was associated with lower HRCT fibrosis scores and was an independent predictor of longer survival time in 96 out of 204 IPF patients. Moreover, the same authors in a subsequent investigation showed that BALF pepsin was elevated in a subgroup of patients with acute exacerbation of IPF, further confirming the major role of microaspiration in IPF patients [32]. However, all 
the aforementioned studies have been performed by means of traditional $\mathrm{pH}$-metry, which limited these investigations to measuring only acid reflux without any information on other chemical types (i.e. weakly acidic reflux) and number of reflux episodes, as well as the risk of proximal migration of the refluxate. Using the current state-of-the-art method to assess GOR (i.e. combined impedance-pH monitoring), we observed that IPF patients had a very severe degree of overall reflux disease, compared not only with non-IPF patients but also compared with those with other respiratory disorders with an established GOR association, such as asthma, cough and laryngitis, as reported in medical literature [33]. Moreover, we showed that IPF patients had higher acid and weakly acidic reflux episodes in both distal and proximal oesophagus, thus favouring the risk of microaspiration into the lungs compared to non-IPF patients. Finding an increased number of weakly acidic reflux episodes is of paramount importance, since this represents a possible explanation of why medical acid suppression alone might fail in preventing reflux and reflux-associated progression of ILD. Moreover, this finding supports the data from the study of LEE et al. [11], who reported that IPF patients who underwent Nissen fundoplication in order to block all reflux had an additional benefit in terms of life survival time. The good correlation we found between the degree of pulmonary fibrosis (HRCT score) and the number of both distal and proximal reflux episodes in IPF patients, as well as the presence/ concentration of pepsin and bile acids in BALF, reinforces the potential role of GOR in the development and/or progression of pulmonary fibrosis. In particular, it is worth noting that the nonsignificant correlation we observed between the degree of pulmonary fibrosis and the number of both distal and proximal reflux episodes in non-IPF patients suggests that abnormal GOR in IPF subjects may not simply be the result of the underlying fibrosis of the lungs, but has the potential to represent a factor unrelated to pulmonary stiffness.

The relevance of both types of reflux was also corroborated by the fact that we documented abnormal levels of pepsin and bile acids in saliva and BALF of a great number of our IPF patients. These data witness the increased risk of pulmonary aspiration of gastric contents (increased bile acids and pepsin in saliva) or the definite evidence of gastric fluid regurgitation and aspiration into the airways (bile acids and pepsin in BALF). To date, although it is generally accepted that the pulmonary aspiration of gastric material may occur in IPF patients, the evidence of this phenomenon is scarce, and mainly based on studies performed in patients with other diseases or conditions [34-36]. Our study is the first evaluating bile acids and pepsin in BALF of IPF patients who concomitantly underwent impedance-pH to correlate reflux findings with specific biomarkers in human fluids. Bile acids were found in BALF of $62 \%$ of IPF patients compared to $25 \%$ of non-IPF patients and $0 \%$ of non-ILD patients, while pepsin was present in $67 \%$ of IPF patients compared to $25 \%$ of non-IPF patients and $0 \%$ of non-ILD patients. These data emphasise the role of aspiration of gastric contents in IPF patients and confirm the high specificity of pepsin and bile salts for diagnosing GOR-associated pulmonary aspiration, as previously reported [36]. Our results also document that $61 \%$ of IPF patients had bile acids in their saliva compared to $33 \%$ of non-IPF patients and $0 \%$ of healthy volunteers, while pepsin was present in $68 \%$ of IPF patients compared to $36 \%$ of non-IPF patients and $0 \%$ of the healthy volunteers. These data allow us to estimate the high risk of pulmonary aspiration of material from the stomach in our IPF patients.

Despite the vast majority of our IPF patients having abnormal GOR, only 48\% (19 out of 40) complained of typical symptoms of GOR, a finding in accordance with previous reports [5, 37]. Therefore, abnormal acid or weakly acidic GOR was often clinically silent. These findings suggest that all ILD patients with or without apparent reflux symptoms should be carefully evaluated and, eventually, should undergo oesophageal impedance-pH to detect asymptomatic GOR. However, we have to stress that further outcome data are necessary to support testing or treatment of IPF patients for abnormal GOR.

As to the limitations of our study, it has been hypothesised that the increased respiratory workload in patients with ILD could contribute to GOR by increasing the transdiaphragmatic pressure gradient [38], thus suggesting that stiffened lungs due to fibrosis may cause associated reflux. Unfortunately, our current methodology (i.e. impedance manometry) did not allow us to assess this parameter. Moreover, we did not find any difference concerning the mean LOS pressure and the prevalence of abnormal motility patterns between the two groups of IPF and non-IPF patients. This finding seems to suggest that the degree of oesophageal motor disturbances does not seem to be associated with the development of pulmonary fibrosis in IPF patients. Therefore, although causality has not been demonstrated, a strong association between IPF and GOR was observed and the good correlation between degree of pulmonary fibrosis (HRCT score) and reflux disease (number of reflux episodes), as well as the presence/concentration of pepsin and bile acids in BALF calls for further investigations in this direction.

In conclusion, current data indicate that IPF patients have greater GOR compared to non-IPF ones. Acid reflux is predominant, but also weakly acidic reflux is increased. IPF patients have a high risk of pulmonary aspiration of gastric contents even in the absence of typical reflux symptoms, and despite evidence of 
normal oesophageal peristalsis. The increased frequency of weakly acidic reflux implies that therapies aimed at reducing overall (and not only acid) reflux should be included in studies aimed at assessing whether or not the development of IPF can be prevented by blocking GOR. Although outcome studies are mandatory to confirm the prominent role of GOR in IPF patients, our data suggest that abnormal GOR should be searched for and treated adequately to prevent micro-aspiration of gastric contents and its potential deleterious effect in the induction, progression, and/or exacerbation of pulmonary fibrosis in patients with IPF.

\section{References}

Idiopathic pulmonary fibrosis: diagnosis and treatment. International consensus statement. Am J Respir Crit Care Med 2000; 161: 646-664.

Ing AJ. Interstitial lung disease and gastroesophageal reflux. Am J Med 2001; 111: Suppl. 8A, 41S-44S.

Savarino E, Bazzica M, Zentilin P, et al. Gastroesophageal reflux and pulmonary fibrosis in scleroderma: a study using pH-impedance monitoring. Am J Respir Crit Care Med 2009; 179: 408-413.

4 Tobin RW, Pope CE 2nd, Pellegrini CA, et al. Increased prevalence of gastroesophageal reflux in patients with idiopathic pulmonary fibrosis. Am J Respir Crit Care Med 1998; 158: 1804-1808.

5 Raghu G, Freudenberger TD, Yang S, et al. High prevalence of abnormal acid gastro-oesophageal reflux in idiopathic pulmonary fibrosis. Eur Respir J 2006; 27: 136-142.

6 Sweet MP, Patti MG, Leard LE, et al. Gastroesophageal reflux in patients with idiopathic pulmonary fibrosis referred for lung transplantation. J Thorac Cardiovasc Surg 2007; 133: 1078-1084.

7 Moran TJ. Experimental aspiration pneumonia. IV. Inflammatory and reparative changes produced by intratracheal injections of autologous gastric juice and hydrochloric acid. AMA Arch Pathol 1955; 60: 122-129.

8 Mays EE, Dubois JJ, Hamilton GB. Pulmonary fibrosis associated with tracheobronchial aspiration. A study of the frequency of hiatal hernia and gastroesophageal reflux in interstitial pulmonary fibrosis of obscure etiology. Chest 1976; 69: 512-515.

9 Raghu G, Yang ST, Spada C, et al. Sole treatment of acid gastroesophageal reflux in idiopathic pulmonary fibrosis: a case series. Chest 2006; 129: 794-800.

10 Linden PA, Gilbert RJ, Yeap BY, et al. Laparoscopic fundoplication in patients with end-stage lung disease awaiting transplantation. J Thorac Cardiovasc Surg 2006; 131: 438-446.

11 Lee JS, Ryu JH, Elicker BM, et al. Gastroesophageal reflux therapy is associated with longer survival in patients with idiopathic pulmonary fibrosis. Am J Respir Crit Care Med 2011; 184: 1390-1394.

12 Downing TE, Sporn TA, Bollinger RR, et al. Pulmonary histopathology in an experimental model of chronic aspiration is independent of acidity. Exp Biol Med (Maywood) 2008; 233: 1202-1212.

13 Mertens V, Blondeau K, Vanaudenaerde B, et al. Gastric juice from patients "on" acid suppressive therapy can still provoke a significant inflammatory reaction by human bronchial epithelial cells. J Clin Gastroenterol 2010; 44: e230-e235.

14 Tutuian R, Castell DO. Use of multichannel intraluminal impedance to document proximal esophageal and pharyngeal nonacidic reflux episodes. Am J Med 2003; 115: Suppl. 3A, 119S-123S.

15 Zentilin P, Dulbecco P, Savarino E, et al. Combined multichannel intraluminal impedance and pH-metry: a novel technique to improve detection of gastro-oesophageal reflux: literature review. Dig Liver Dis 2004; 36: 565-569.

16 Statement on sarcoidosis. Joint Statement of The American Thoracic Society (ATS), the European Respiratory Society (ERS) and the World Association of Sarcoidosis and Other Granulomatosous Disorders (WASOG) adopted by the ATS Board of Directors and by the ERS Executive Committee, February 1999. Am J Respir Crit Care Med 1999; 160: 736-755.

17 Kallenberg CG. Overlapping syndromes, undifferentiated connective tissue disease, and other fibrosing conditions. Curr Opin Rheumatol 1995; 7: 568-573.

18 Tan EM, Cohen AS, Fries JF, et al. The 1982 revised criteria for the classification of systemic lupus erythematosus. Arthritis Rheum 1982; 25: 1271-1277.

19 American Thoracic Society/European Respiratory Society. American Thoracic Society/European Respiratory Society international multidisciplinary consensus classification of the idiopathic interstitial pneumonias. Am J Respir Crit Care Med 2002; 165: 277-304.

20 Savarino E, Tutuian R. Combined multichannel intraluminal impedance and manometry testing. Dig Liver Dis 2008; 40: 167-173.

21 Spechler SJ, Castell D. Classification of oesophageal motility abnormalities. Gut 2001; 49: 145-151.

22 Savarino E, Tutuian R, Zentilin P, et al. Characteristics of reflux episodes and symptom association in patients with erosive esophagitis and nonerosive reflux disease: study using combined impedance-pH off therapy. Am J Gastroenterol 2010; 105: 1053-1061.

23 Sifrim D, Castell D, Dent J, et al. Gastro-oesophageal reflux monitoring: review and consensus report on detection and definitions of acid, non-acid, and gas reflux. Gut 2004; 53: 1024-1031.

24 Savarino E, Marabotto E, Zentilin P, et al. The added value of impedance-pH monitoring to Rome III criteria in distinguishing functional heartburn from non-erosive reflux disease. Dig Liver Dis 2011; 43: 542-547.

25 Savarino E, Pohl D, Zentilin P, et al. Functional heartburn has more in common with functional dyspepsia than with non-erosive reflux disease. Gut 2009; 58: 1185-1191.

26 Zentilin P, Iiritano E, Dulbecco P, et al. Normal values of 24-h ambulatory intraluminal impedance combined with pH-metry in subjects eating a Mediterranean diet. Dig Liver Dis 2006; 38: 226-232.

27 Quanjer PH, Tammeling GJ, Cotes JE, et al. Lung volumes and forced ventilatory flows. Report Working Party Standardization of Lung Function Tests, European Community for Steel and Coal. Official Statement of the European Respiratory Society. Eur Respir J 1993; 6: Suppl. 16, 5-40.

28 Cotes JE, Chinn DJ, Quanjer PH, et al. Standardization of the measurement of transfer factor (diffusing capacity). Report Working Party Standardization of Lung Function Tests, European Community for Steel and Coal. Official Statement of the European Respiratory Society. Eur Respir J 1993; 6: Suppl. 16, 41-52.

29 Best AC, Meng J, Lynch AM, et al. Idiopathic pulmonary fibrosis: physiologic tests, quantitative CT indexes, and CT visual scores as predictors of mortality. Radiology 2008; 246: 935-940. 

with cystic fibrosis. Gut 2008; 57: 1049-1055.

31 Fahim A, Dettmar PW, Morice AH, et al. Gastroesophageal reflux and idiopathic pulmonary fibrosis: a prospective study. Medicina (Kaunas) 2011; 47: 200-205.

32 Lee JS, Song JW, Wolters PJ, et al. Bronchoalveolar lavage pepsin in acute exacerbation of idiopathic pulmonary fibrosis. Eur Respir J 2012; 39: 352-358.

33 Ahmed T, Vaezi MF. The role of $\mathrm{pH}$ monitoring in extraesophageal gastroesophageal reflux disease. Gastrointest Endosc Clin N Am 2005; 15: 319-331.

34 Knight J, Lively MO, Johnston N, et al. Sensitive pepsin immunoassay for detection of laryngopharyngeal reflux. Laryngoscope 2005; 115: 1473-1478.

35 Stovold R, Forrest IA, Corris PA, et al. Pepsin, a biomarker of gastric aspiration in lung allografts: a putative association with rejection. Am J Respir Crit Care Med 2007; 175: 1298-1303.

36 Farrell S, McMaster C, Gibson D, et al. Pepsin in bronchoalveolar lavage fluid: a specific and sensitive method of diagnosing gastroesophageal reflux-related pulmonary aspiration. J Pediatr Surg 2006; 41: $289-293$.

37 Pellegrini CA, DeMeester TR, Johnson LF, et al. Gastroesophageal reflux and pulmonary aspiration: incidence, functional abnormality, and results of surgical therapy. Surgery 1979; 86: 110-119.

38 Brennan NJ, Morris AJR, Green M. Thoracoabdominal mechanics during tidal breathing in normal subjects and in emphysema and fibrosing alveolitis. Thorax 1983; 38: 62-66. 